\title{
TIME-DOMAIN OBSERVATION AND SYNTHESIS OF SPLIT SPHEROIDAL AND TORSIONAL FREE OSCILLATIONS OF THE 1960 CHILEAN EARTHQUAKE: PRELIMINARY RESULTS
}

\author{
By Seth Stein and Robert J. Geller*
}

\begin{abstract}
The rotationally and elliptically split normal modes of the earth are observed for the 1960 Chilean earthquake by analysis in the time domain. One hundred and fifty hours of the Isabella, California, strain record are narrow band filtered about the central frequency of each split multiplet to isolate the complex wave form resulting from the interference of the different singlets. We compute synthetic seismograms using our previous theoretical results, which show the dependence of the amplitude and phase of the singlets on source location, depth, mechanism, and the position of the receiver. By comparing these synthetics to the filtered record, we conclusively demonstrate the splitting of modes whose splitting had not been definitely resolved: torsional modes $\left(0 T_{3}, 0 T_{4}\right)$ and spheroidal modes $\left({ }_{0} S_{4},{ }_{0} S_{5}\right)$. The splitting of ${ }_{0} S_{2}$ and ${ }_{0} S_{3}$ is reconfirmed. We obtain good agreement between the synthetics and the filtered data for a source mechanism (previously determined from long-period surface waves) of thrust motion on a shallow dipping fault.
\end{abstract}

\section{INTRODUCTION}

Split peaks in the earth's normal mode spectrum were first observed for the 1960 Chilean earthquake. The splitting of ${ }_{0} S_{2}$ and ${ }_{0} S_{3}$ was reported by Benioff, Press, and Smith (1961) and Ness, Harrison, and Slichter (1961). Slichter (1967) observed the splitting of ${ }_{0} S_{3}$ for the 1964 Alaskan earthquake. Splitting has never been conclusively demonstrated for other spheroidal modes or for any torsional modes.

Extensive theoretical efforts (summarized in our earlier papers) have been devoted to the computation of the eigenfrequencies of the $2 l+1$ individual singlets into which the multiplet of angular order $l$ (e.g., ${ }_{n} S_{l}$ ) is split. This frequency splitting of the very long-period modes is primarily due to the earth's rotation (Pekeris et al., 1961 and Backus and Gilbert, 1961) and ellipticity (Dahlen, 1968). In an earlier paper (Stein and Geller, 1977) we derived theoretical results that allow us to calculate the amplitudes and phases of the split normal modes (or equivalently, their time history) excited by a double couple of arbitrary orientation resulting from slip on a fault plane. These results were applied successfully (Geller and Stein, 1977) to split spectra of the Chilean and Alaskan earthquakes.

In this paper we use source models derived from long-period surface wave studies to generate synthetic seismograms for each of six multiplets $\left({ }_{0} S_{2}-{ }_{0} S_{5},{ }_{0} T_{3},{ }_{0} T_{4}\right)$, and compare them to a time-domain record of the Chilean earthquake which has been narrow band filtered to isolate that multiplet. This procedure yields several results which were not obtained by earlier studies in the frequency domain. We show that splitting is present for modes for which it has not been previously resolved, including torsional modes.

Our results are derived entirely from wave-form shapes, and make no use of absolute amplitude information, due to calibration complexities. The absolute amplitudes are used in a forthcoming study (Stein and Geller, 1978) to determine the moment at very long periods. We also determine accurate $Q$ 's for the low-order modes.

* Also Department of Geophysics, Stanford University, Stanford, California 94305. 


\section{DATA AND ANALYSIS}

Figure 1 shows $150 \mathrm{hr}$ of the strain record (filtered electronically to reduce the tidal amplitudes) from Isabella, California (Benioff et al., 1961) for the great 1960 Chilean earthquake. The record (top) was processed to remove tides by twice subtracting 3 -h running averages. The resulting record (bottom) was tapered at both ends and then filtered to isolate and resolve individual low angular order modes. The resulting narrow band filtered data are shown in the upper traces of Figures 2 through 7, for four spheroidal and two torsional modes. All these wave forms show the general $\left(e^{-w t / 2 Q}\right)$ attenuation of the entire mode superimposed on a complicated pattern which results from the interference of the split singlets.

The middle trace of each figure shows a synthetic seismogram for each mode which includes the effects of splitting. The results of Stein and Geller (1977) for

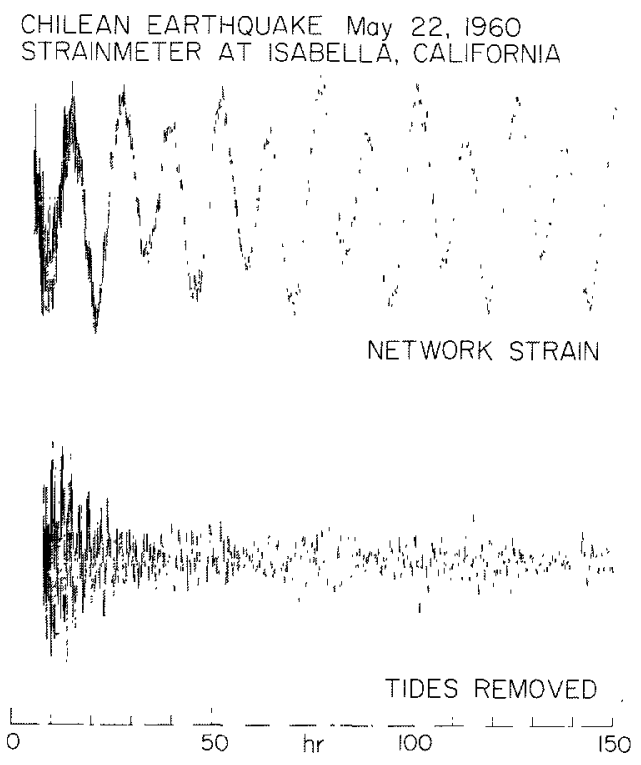

FIG. 1. Isabella strain record of the Chilean earthquake (top trace) and the high-passed record with tides removed (bottom trace). The origin time of this figure and all others, is 1911 hours, 22 May 1960, the origin time of the main shock. The digitized record (top) begins $289 \mathrm{~min}$ later, at $0000 \mathrm{hr}, 23 \mathrm{May}$. The high-passed record (bottom) starts another $3 \mathrm{hr}$ later.

the excitation of split modes are used to calculate the synthetics. The effect of splitting can be seen by examining the lower traces, which are calculated without splitting. In this case each mode appears as a pure damped harmonic oscillator, since all its singlets have the same frequency. The data are fit far better by the synthetics with splitting than by those without it, and thus splitting is demonstrated.

The eigenfrequency of each singlet is computed using Anderson and Hart's (1978) values for the unperturbed eigenfrequencies and Dahlen's (1968) rotational splitting parameters. The elliptical splitting parameters are not used, due to the difficulties involved in previous calculations of these parameters (Woodhouse, 1976). Dahlen (personal communication) states that more accurate recent computations show that the spheroidal mode elliptical splitting parameters may be neglected for our purposes. Accurate values have not yet been published for the elliptical splitting of torsional modes, or for splitting due to lateral heterogeneities.

Synthetic seismograms are computed for a range of source parameters; good agreement is obtained for the source mechanism determined by Kanamori and 
Cipar (1974) from long-period surface waves. The rupture was initiated at $38^{\circ} \mathrm{S}$, $286.5^{\circ} \mathrm{E}$ and propagated at $3.5 \mathrm{~km} / \mathrm{sec}$ to $46^{\circ} \mathrm{S}, 286.5^{\circ} \mathrm{E}$, on a fault plane dipping $10^{\circ}$ east and striking $\mathrm{N} 10^{\circ} \mathrm{E}$. (We approximate the finite source by five point sources at a depth of $55 \mathrm{~km}$.) The slip angle is $90^{\circ}$, a pure thrust motion. We are also including a precursory slip (Kanamori and Cipar, 1974; Kanamori and Anderson, 1975 ) at $41.5^{\circ} \mathrm{S}, 285.7^{\circ} \mathrm{E}$ with a rise time of $5 \mathrm{~min}$ starting $15 \mathrm{~min}$ before the main shock, and with a moment equal to that of the main shock.

Excellent agreement between the synthetics and data is obtained for the longest period spheroidal modes, ${ }_{0} S_{2}$ and ${ }_{0} S_{3}$ (Figures 2 and 3 ). The absolute amplitude of

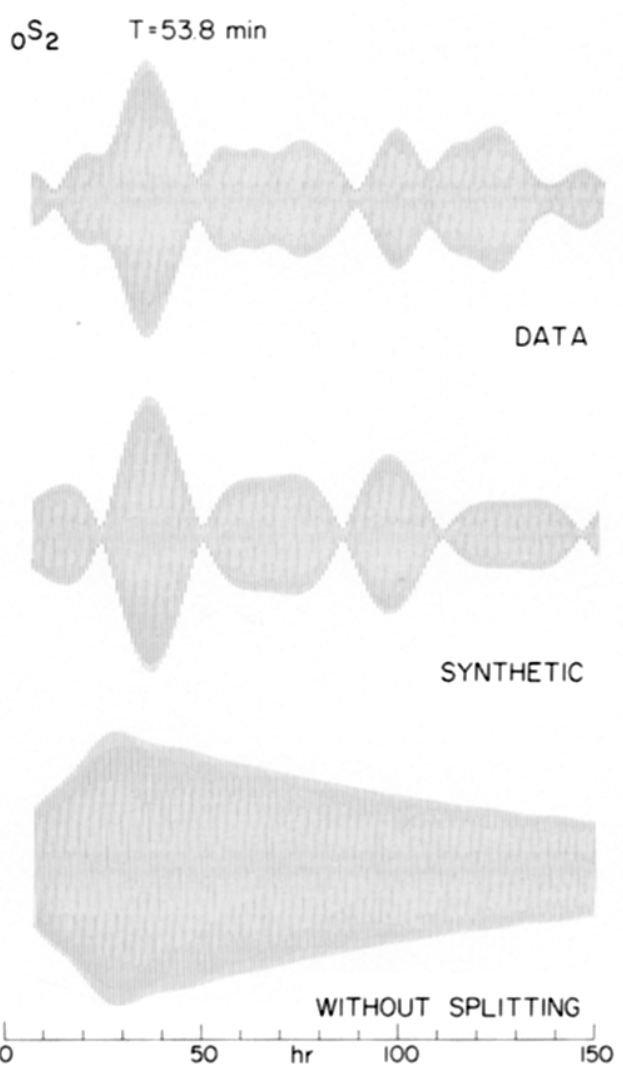

FIG. 2. Data and synthetics for ${ }_{0} S_{2}$. The top trace is filtered data from the high-passed Isabella strain record of the Chilean earthquake. The middle trace is the synthetic seismogram, including the effects of splitting, and the bottom trace is the synthetic without splitting. Both synthetics are computed using the same $Q$. The synthetics were tapered and filtered in the same way as the data. The filtering and tapering are responsible for reducing the initial amplitude (especially visible in the bottom trace).

these modes on the Isabella record is about three times greater than any of the other modes (Geller, 1977). Thus the general envelope shape of these modes match the data quite well. Note the large peak at $35 \mathrm{hr}$ for ${ }_{0} S_{2}$ (Figure 2) and the peak at $80 \mathrm{hr}$ for ${ }_{0} S_{3}$ (Figure 3). The nodal times of the data and synthetics also agree quite well.

We obtain acceptable fits for the other modes. Their absolute trace amplitudes are much lower, and in the case of ${ }_{0} T_{3},{ }_{0} T_{4}$ and ${ }_{0} S_{4}$ and ${ }_{0} S_{5}$ are barely resolvable above the noise (Benioff et al., 1961). Nonetheless, the beat pattern is clearly evident (Figures 4 to 7), demonstrating splitting. The discrepancies between the data and synthetics are probably due to the noise, and, in the case of torsional modes, 
ellipticity splitting. Given these difficulties, the agreement of data and synthetics is quite acceptable.

We tested a wide variety of source geometries and obtained good results for the source parameters given previously. We thus conclude that the fault mechanism derived at periods of several hundred seconds is consistent with the data at far longer periods. This does not, of course, demonstrate that this is the only acceptable source mechanism. A more accurate mechanism might result from use of better splitting parameters, but will still be limited primarily by the noise in the data.

A prior study of split spectra (Smith, 1961) suggested the possible splitting of ${ }_{0} S_{4}$,
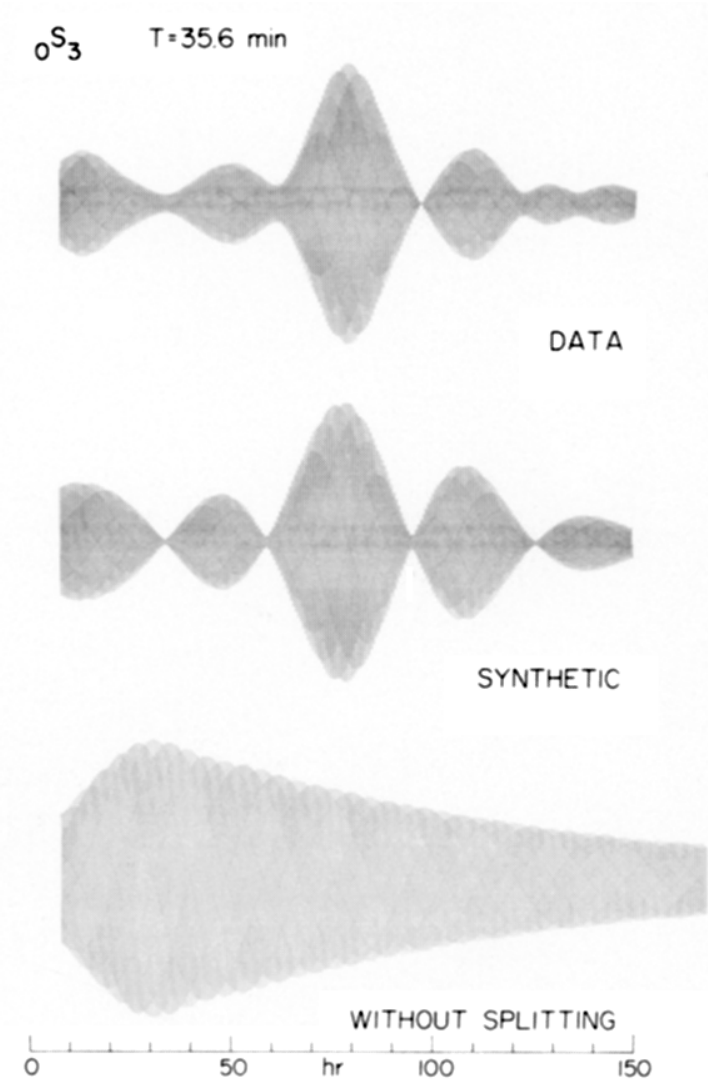

FIG. 3. Data and synthetics for $n S_{3}$. Details are as in Figure 2. Note that the maximum trace amplitude occurs $80 \mathrm{hr}$ into the record.

${ }_{0} T_{2}$, and ${ }_{0} T_{3}$, but Smith noted that the data for these modes were marginal. The high resolution spectra presented by Wiggins and Miller (1972) for the UCLA gravity record of the 1964 Alaskan earthquake also suggest the splitting of $S_{4}$. Our time domain analysis shows the beat pattern, and thus the splitting, of ${ }_{0} S_{4},{ }_{0} S_{5},{ }_{0} T_{3}$, and ${ }_{0} T_{4}$.

\section{Apparent $\boldsymbol{Q}$ of Split Modes}

We study the attenuation of split modes by comparing the data to the synthetics. Splitting is no longer visible when the beat time is longer than the $Q$ decay time. [In the frequency domain, this occurs when the broadening of spectral peaks due to 
attenuation is much greater than the frequency separation of singlets resulting from rotation and ellipticity (Gilbert and Backus, 1965).] Figures 5 and 7, respectively, show that splitting is barely detectable for ${ }_{0} S_{5}$ and ${ }_{0} T_{4}$, because the synthetics with and without splitting are much more similar than those for the longer period modes.

The synthetics are computed by using a $Q$ of 400 in Figures 2, 4, and 5; a $Q$ of 300 for Figure 3; and a $Q$ of $\mathbf{4 5 0}$ for Figures 6 and 7. These $Q$ values are nominal and cannot be well constrained by using only $150 \mathrm{hr}$ of data.

It is important to note that because the amplitude of the record does not decrease monotonically with time, it is difficult to determine $Q$ by examining the amplitude of spectral peaks from successive time windows. This is demonstrated by ${ }_{0} S_{3}$ (Figure
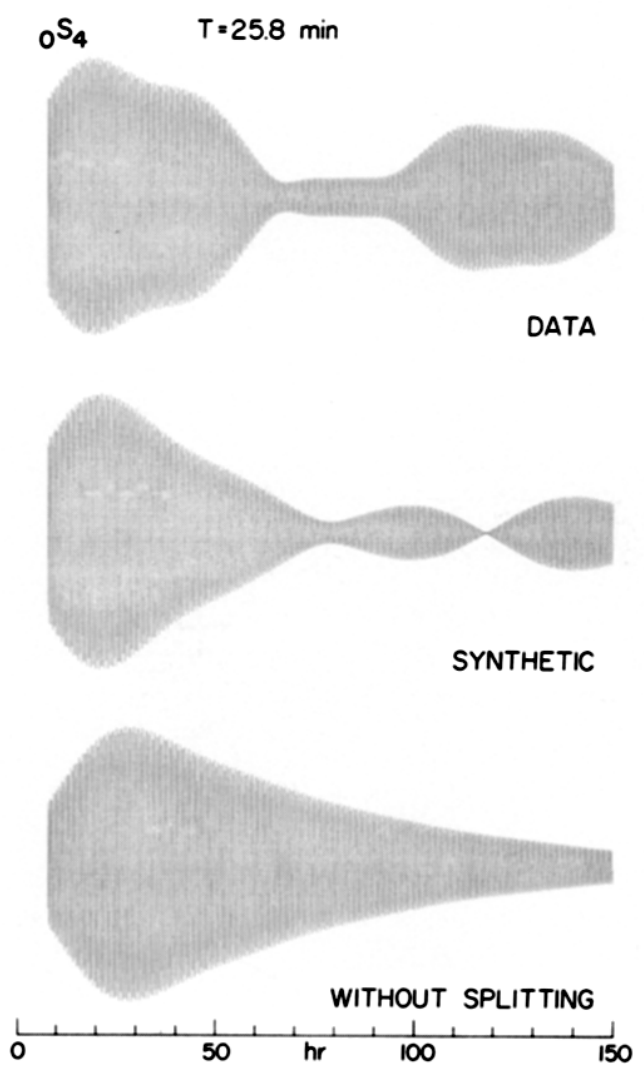

FIG. 4. Data and synthetics for ${ }_{0} S_{4}$. Details are as in Figure 2. Substantial noise appears to be present in the data.

3 ), in which the maximum trace amplitude occurs $80 \mathrm{hr}$ into the record. Thus the peak spectral amplitude will not even be a monotonically decaying function of time.

A more subtle difficulty in amplitude decay analysis occurs even when beats are not apparent on the filtered seismogram. For these long-period modes, the amplitude as a function of time is controlled at least as much by the interference of the singlets as by attenuation. This can be seen clearly by comparing the decay of the two synthetics for ${ }_{0} S_{5}$ (Figure 5), computed with the same $Q$. Destructive interference due to splitting makes the synthetic with splitting (middle trace) decay much 

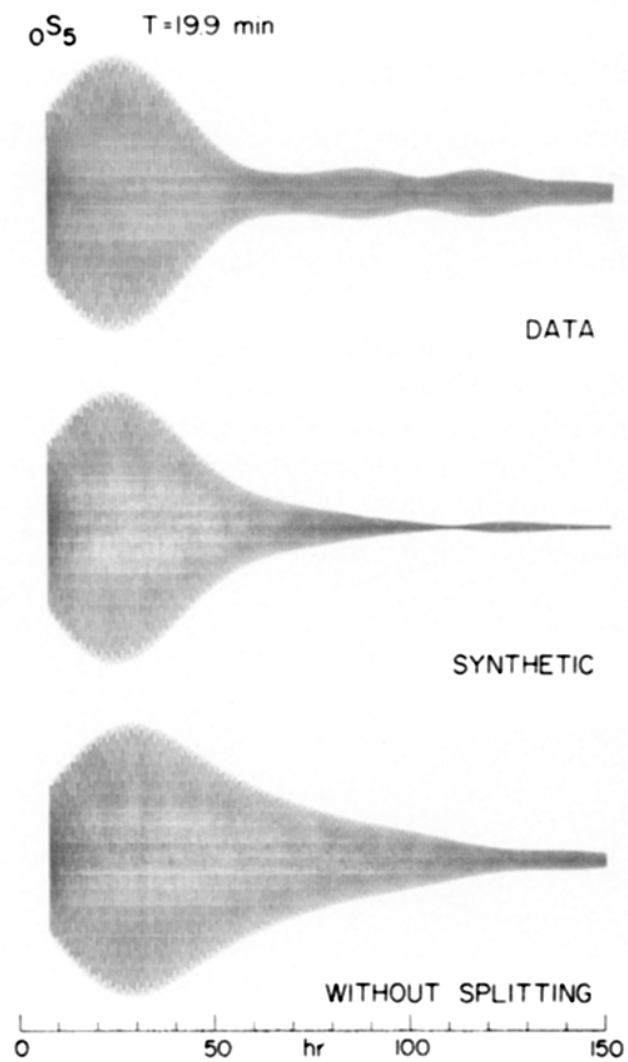

FIG. 5. Data and synthetics for ${ }_{0} S_{5}$. Details are as in Figure 2. The two synthetics computed with the same $Q$ decay at different rates as a result of the destructive interference caused by splitting.
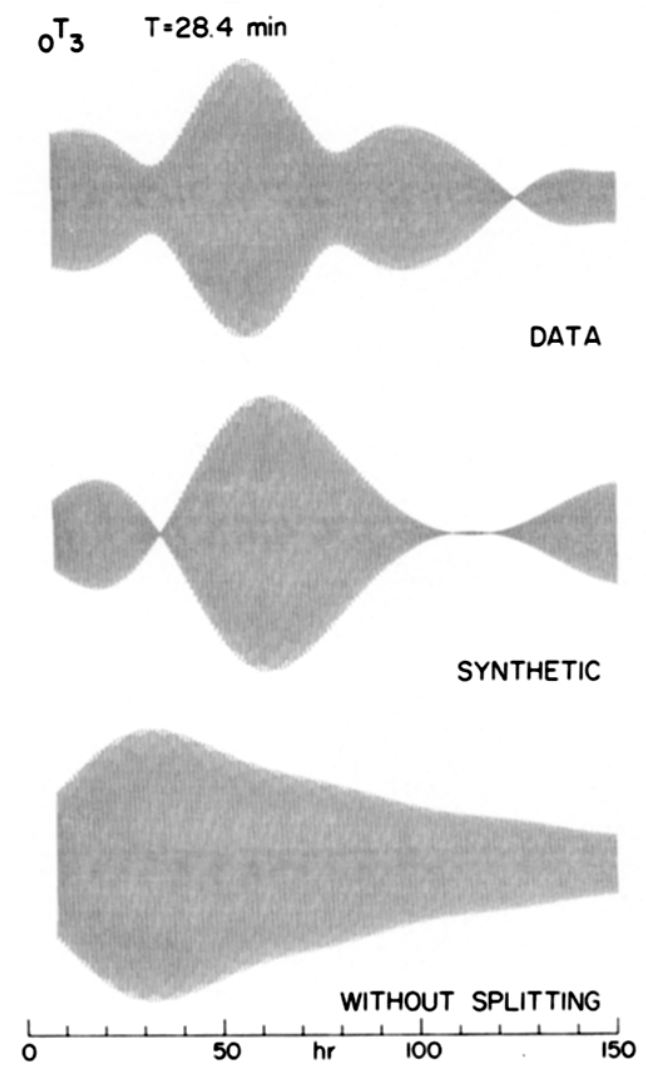

FIG. 6. Data and synthetics for ${ }_{0} T_{3}$. Details are as in Figure 2. 
faster than the unsplit synthetic (bottom trace). Thus if splitting is neglected, the $Q$ measured from the amplitude decay would be far too low.

It is thus clear that splitting must be correctly included for accurate $Q$ determinations. Such determinations are difficult and beyond the scope of this paper. Our intent here is to discuss the importance of splitting for the measurement of the $Q$ 's of long-period modes.

Alsop et al. (1961) discuss the measurement of $Q$ for split modes. They show that $Q$ can be correctly measured under several conditions: either if the record length is adequate to completely resolve individual singlets, or the record length is much greater than the beat time, or if the same portion of a cycle is being averaged over. Our method is applicable in general even if these special circumstances are not fulfilled.



Fig. 7. Data and synthetics for ${ }_{0} T_{4}$. Details are as in Figure 2 .

\section{CONCLUSION}

The comparison of narrow band filtered records to computed synthetic seismograms provides a new method for the analysis of normal modes when splitting is present. This technique allows the resolution of splitting in cases when spectral amplitude analysis (which does not incorporate phase information) is insufficient. We confirm the splitting of ${ }_{0} S_{2}$ and ${ }_{0} S_{3}$, and demonstrate the splitting of ${ }_{0} S_{4}, 0 S_{5}, 0 T_{3}$, and ${ }_{0} T_{4}$. Time domain analysis of split normal modes represents a powerful new approach to ultra-long period source and attenuation studies. 


\section{ACKNOWLEDGMENTS}

We thank Stewart Smith and Hiroo Kanamori for providing us with data from and information about the Isabella strain record. Yoshio Fukao, Hiroo Kanamori, and Emile Okal critically read the manuscript. We have also benefited from the comments of an anonymous reviewer. Seth Stein was supported by a fellowship from the Fannie and John Hertz Foundation. This research was supported by the Division of Earth Sciences, National Science Foundation, NSF Grants EAR76-14262, EAR74-22489, and EAR77-14675.

\section{REFERENCES}

Alsop, L. E., G. H. Sutton, and M. Ewing (1961). Measurement of Q for very long period free oscillations, J. Geophys. Res. 66, 2911-2915.

Anderson, D, L. and R. S. Hart (1977). The Q of the Earth, J. Geophys. Res. (in press).

Backus, G. and F. Gilbert (1961). The rotational splitting of the free oscillations of the Earth, Proc. Natl. Acad. Sci. U.S. 47, 362-371.

Benioff, H., F. Press, and S. Smith (1961). Excitation of the free oscillations of the Earth by earthquakes, J. Geophys. Res. 66, 605-619.

Dahlen, F. A. (1968). The normal modes of a rotating, elliptical Earth, Geophys. J. 16, 329-367.

Geller, R. J. (1977). Amplitudes of rotationally split normal modes for the 1960 Chilean and 1964 Alaskan earthquakes, Ph.D. Thesis (Part II), California Institute of Technology.

Geller, R. J. and S. Stein (1977). Split free oscillation amplitudes for the 1960 Chilean and 1964 Alaskan earthquakes, Bull. Seism. Soc. Am. 67, 651-660.

Gilbert, F. and G. Backus (1965). The rotational splitting of the free oscillations of the Earth, 2, Rev. Geophys. 3, 1-9.

Kanamori, H. and D. L. Anderson (1975). Amplitude of the Earth's free oscillations and long period characteristics of the earthquake source, J. Geophys. Res. 80, 1075-1078.

Kanamori, H. and J. J. Cipar (1974). Focal process of the great Chilean earthquake, May 22, 1960, Phys. Earth. Planet Interiors 9, 128-136.

Ness, N., J. Harrison, and L. Slichter (1961). Observations of the free oscillations of the earth, J. Geophys. Res. 66, 621-629.

Pekeris, C. L., Z. Alterman, and H. Jarosch (1961). Rotational multiplets in the spectrum of the Earth, Phys. Rev. 122, 1692-1700.

Slichter, L. B. (1967). Spherical oscillations of the Earth, Geophys. J. 14, 171-177.

Smith, S. W. (1961). An investigation of the Earth's free oscillations, Ph.D. Thesis, California Institute of Technology.

Stein, S. and R. J. Geller (1977). Amplitudes of the split normal modes of a rotating, elliptical Earth excited by a double couple, J. Phys. Earth, 25, 117-142.

Stein, S. and R. J. Geller (1978). Attenuation measurements of split normal modes for the 1960 Chilean and 1964 Alaskan earthquakes (to be submitted for publication).

Wiggins, R. A. and S. P. Miller (1972). New noise-reduction technique applied to long-period oscillations from the Alaskan earthquake, Bull. Seism. Soc. Am. 62, 471-479.

SEISMOLOGICAL LABORATORY

California Institute of Technology

Pasadena, California 91125

CONTRIBUTion No. 2892

Manuscript received May 24, 1977 